\title{
An Analytical Model of Trilateration Localization Error
}

\author{
Muhammad Farooq-I-Azam ${ }^{1}$, Qiang $\mathrm{Ni}^{2}$ and Mianxiong Dong ${ }^{3}$ \\ ${ }^{1}$ Department of Electrical \& Computer Engineering, COMSATS University Islamabad, Lahore Campus, Pakistan. \\ ${ }^{2}$ InfoLab21, School of Computing \& Communications, Lancaster University, UK. \\ ${ }^{3}$ Department of Information and Electronic Engineering, Muroran Institute of Technology, Japan.
}

\begin{abstract}
Trilateration and multilateration are important location estimation techniques used in a diverse range of networks and applications. The system of equations yielded by multilateration can be reduced to simpler linear equations which can be solved to arrive at a closed form analytic solution. Exploiting this solution technique, we develop a novel and unique analytical model for the localization error resulting from trilateration. The analytical model can be used for the analysis of the localization error in all applications wherever multilateration is used for position estimation including internet of things, wireless sensor networks and global navigation satellite system thereby increasing reliability and quality of localization. As an example, we use the analytical model to corroborate the fact that localization error is a function of topology of reference positions in addition to distance estimation errors. The analytical model is verified using simulation experiments.
\end{abstract}

\section{INTRODUCTION}

Location information is important in many disciplines of science and engineering. For example, in many applications of Internet of things (IoT) and wireless sensor networks (WSN), data are ascribed to the locations from where these are gathered. For this to be possible, a randomly deployed sensor or IoT node should be able to estimate its position [1], [2] using a localization algorithm [3]. Location information can also be used to improve quality of service and reliability in communication systems. For example, spectrum sensing is an important technique and enabling factor for dynamic spectrum sharing in future $5 \mathrm{G}$ communications. Location estimation of the primary user results in reliable spectrum sensing and cognitive enhancement [4]. Likewise, location information can be used for subcarrier and power allocation in a cognitive radio network [5] for better and efficient communication. Due to its significance, location awareness is included in the six important cognitive abilities of a cognitive radio network identified by Federal Communication Commission (FCC) [6]. In context aware and pervasive computing, context or location information of a user is used for the adaption and provision of computing services without explicit intervention of the user [7]. In space and moon exploration missions, an unmanned rover should be able to estimate its position so that it can continue towards its desired destination [8].

Within the localization domain, trilateration and multilateration are important techniques to estimate position of an object given its distances to known positions. For estimation of position in two dimensions, distances to three

This research was supported in part by the Royal Society project IEC170324, EPSRC project EP/K011693/1, EPSRC IAA project CSA7113, EU FP7 CROWN project PIRSES GA-2013-610524, JSPS KAKENHI Grant JP16K00117 and the KDDI Foundation. or more known locations are required. In the case of three dimensional position estimation, distance estimates to four or more locations with known position coordinates are required. In the literature, the terms, trilateration and multilateration have been used in various contexts. In our work, we use the term trilateration when the distance estimates to only three known positions in two dimensions or four known positions in three dimensions are used. For estimation of position using distance estimates to higher number of reference positions, we use the term multilateration.

Main contribution of our work is the development of an analytical model of trilateration localization error. Using the multilateration solution, we give an exact, analytic and deterministic model of localization error as a function of distance estimation errors and positions of reference nodes.

- The model establishes the relationship between localization error, the distance estimation errors and the topology of the beacon nodes.

- Furthermore, it isolates the localization error from the estimated position and decomposes it into various components. This allows treatment of localization error by using various mathematical techniques and tools.

- The analytical model can be used for all the applications wherever multilateration and trilateration are used for the estimation of position.

- The model can be used for the analysis and reduction of trilateration localization error in these applications.

To the best of our knowledge, this paper is the first attempt to present an exact, analytic and deterministic model of trilateration localization error. It provides a novel avenue to explore localization error and hence advances state of the art. We perform a number of simulation experiments to test the analytical model. ${ }^{1}$ The results verify our proposed model.

\section{Multilateration Solution}

In this section, we provide background information and describe the multilateration solution approach that we subsequently exploit to develop our analytical model of trilateration localization error.

To explain position estimation and to investigate multilateration solution, consider an unknown node with actual position coordinates $\left(x_{a}, y_{a}\right)$ having $k$ neighbor beacon nodes with position coordinates $\left(x_{1}, y_{1}\right),\left(x_{2}, y_{2}\right), \ldots,\left(x_{k}, y_{k}\right)$. If estimated position of the unknown node is $(x, y)$, then a set

\footnotetext{
${ }^{1}$ The complete data set resulting from the simulation experiments is available at IEEE DataPort at http://dx.doi.org/10.21227/9bk9-y425
} 
of the following equations of circles around the beacon nodes can be obtained by using the position coordinates of the beacon nodes as centers and the range estimates $r_{1}, r_{2}, \ldots, r_{k}$ between the unknown node and the beacon nodes as radii:

$$
\left[\begin{array}{c}
\left(x-x_{1}\right)^{2}+\left(y-y_{1}\right)^{2} \\
\left(x-x_{2}\right)^{2}+\left(y-y_{2}\right)^{2} \\
\vdots \\
\left(x-x_{k}\right)^{2}+\left(y-y_{k}\right)^{2}
\end{array}\right]=\left[\begin{array}{c}
r_{1}^{2} \\
r_{2}^{2} \\
\vdots \\
r_{k}^{2}
\end{array}\right]
$$

This can be manipulated, rearranged and written in matrix form as below,

$$
\mathbf{A z}=\mathbf{R}
$$

where $\mathbf{z}=\left[\begin{array}{ll}x & y\end{array}\right]^{T}$ and

$$
\begin{gathered}
\mathbf{A}=\left[\begin{array}{cc}
\left(x_{k}-x_{1}\right) & \left(y_{k}-y_{1}\right) \\
\left(x_{k}-x_{2}\right) & \left(y_{k}-y_{2}\right) \\
\vdots & \vdots \\
\left(x_{k}-x_{k-1}\right) & \left(y_{k}-y_{k-1}\right)
\end{array}\right] \\
\mathbf{R}=\frac{1}{2}\left[\begin{array}{c}
r_{1}^{2}-r_{k}^{2}+x_{k}^{2}-x_{1}^{2}+y_{k}^{2}-y_{1}^{2} \\
r_{2}^{2}-r_{k}^{2}+x_{k}^{2}-x_{2}^{2}+y_{k}^{2}-y_{2}^{2} \\
\vdots \\
r_{k-1}^{2}-r_{k}^{2}+x_{k}^{2}-x_{k-1}^{2}+y_{k}^{2}-y_{k-1}^{2}
\end{array}\right] .
\end{gathered}
$$

As matrix $\mathbf{A}$ is not necessarily square, we cannot use $\mathbf{z}=\mathbf{A}^{-\mathbf{1}} \mathbf{R}$ to determine $\mathbf{z}$. However, solution to (2) can be arrived at using either least squares approximation or by an alternate algebraic method. For example, multiplying both sides of (2) by $\mathbf{A}^{\mathbf{T}}$, we get

$$
\mathbf{A}^{\mathbf{T}} \mathbf{A z}=\mathbf{A}^{\mathbf{T}} \mathbf{R}
$$

We can represent (5) in simplified form as below:

$$
\mathbf{H z}=\mathbf{T},
$$

where $\mathbf{H}=\mathbf{A}^{\mathbf{T}} \mathbf{A}$ and $\mathbf{T}=\mathbf{A}^{\mathbf{T}} \mathbf{R}$. It is to be noted that the matrix $\mathbf{A}$ has an order of $(k-1) \times 2$ if the unknown node has $k$ neighbor beacon nodes. Consequently, the order of matrix $\mathbf{A}^{\mathbf{T}}$ is $2 \times(k-1)$. Hence, the order of the resultant matrix $\mathbf{H}=\mathbf{A}^{\mathbf{T}} \mathbf{A}$ is $2 \times 2$. Order of the column matrix $\mathbf{z}$ is $2 \times 1$. Hence matrices $\mathbf{H}$ and $\mathbf{z}$ are conformable for multiplication. Similarly, the matrix $\mathbf{T}=\mathbf{A}^{\mathbf{T}} \mathbf{R}$ has an order of $2 \times 1$. In other words, (6) represents a system of two linear equations which can be solved simultaneously using conventional techniques. We choose to localize an unknown node only when it has three or more neighbor beacon nodes. Therefore, $k-1 \geq 2$ i.e. number of rows in matrix $\mathbf{A}$ is always equal to or greater than the number of columns. This gives us an exact solution if it exists and an approximate solution otherwise. The matrix $\mathbf{A}$ is singular and the solution does not exist when all the neighbor beacon nodes of an unknown node are collinear.

\section{ANALYTiCAL MODEL}

Let the estimated distances between the unknown node and the $k$ neighbor beacon nodes be $r_{1}, r_{2}, \ldots, r_{k}$ and the actual distances be $r_{a 1}, r_{a 2}, \ldots, r_{a k}$. The estimated distances may have errors $e_{1}, e_{2}, \ldots, e_{k}$ due to factors, such as channel impairments if the positions are estimated using signal strength measurements. Therefore,

$$
\left[\begin{array}{c}
r_{1} \\
r_{2} \\
\vdots \\
r_{k}
\end{array}\right]=\left[\begin{array}{c}
r_{a 1}+e_{1} \\
r_{a 2}+e_{2} \\
\vdots \\
r_{a k}+e_{k}
\end{array}\right] .
$$

Hence, (2) can be rewritten as

$$
\begin{aligned}
& {\left[\begin{array}{cc}
\left(x_{k}-x_{1}\right) & \left(y_{k}-y_{1}\right) \\
\left(x_{k}-x_{2}\right) & \left(y_{k}-y_{2}\right) \\
\vdots & \vdots \\
\left(x_{k}-x_{k-1}\right) & \left(y_{k}-y_{k-1}\right)
\end{array}\right]\left[\begin{array}{c}
x \\
y
\end{array}\right]} \\
& =\frac{1}{2}\left[\begin{array}{cc}
\left(r_{a 1}+e_{1}\right)^{2}-\left(r_{a k}+e_{k}\right)^{2} & +x_{k}^{2}-x_{1}^{2} \\
& +y_{k}^{2}-y_{1}^{2} \\
\left(r_{a 2}+e_{2}\right)^{2}-\left(r_{a k}+e_{k}\right)^{2} & +x_{k}^{2}-x_{2}^{2} \\
\vdots & +y_{k}^{2}-y_{2}^{2} \\
\vdots & \\
\left(r_{a k-1}+e_{a k-1}\right)^{2}-\left(r_{a k}+e_{k}\right)^{2} & +x_{k}^{2}-x_{k-1}^{2} \\
& +y_{k}^{2}-y_{k-1}^{2}
\end{array}\right]
\end{aligned}
$$

Let us now consider a simple case where an unknown node has three neighbor beacon nodes so that $k=3$ and for this specific case (8) becomes:

$$
\begin{aligned}
& {\left[\begin{array}{ll}
\left(x_{3}-x_{1}\right) & \left(y_{3}-y_{1}\right) \\
\left(x_{3}-x_{2}\right) & \left(y_{3}-y_{2}\right)
\end{array}\right]\left[\begin{array}{l}
x \\
y
\end{array}\right]} \\
& =\frac{1}{2}\left[\begin{array}{l}
\left(r_{a 1}+e_{1}\right)^{2}-\left(r_{a 3}+e_{3}\right)^{2}+x_{3}^{2}-x_{1}^{2}+y_{3}^{2}-y_{1}^{2} \\
\left(r_{a 2}+e_{2}\right)^{2}-\left(r_{a 3}+e_{3}\right)^{2}+x_{3}^{2}-x_{2}^{2}+y_{3}^{2}-y_{2}^{2}
\end{array}\right] .
\end{aligned}
$$

As explained in Section II, this can be solved using conventional techniques. Let

$$
\begin{aligned}
\Delta & =\left|\begin{array}{ll}
\left(x_{3}-x_{1}\right) & \left(y_{3}-y_{1}\right) \\
\left(x_{3}-x_{2}\right) & \left(y_{3}-y_{2}\right)
\end{array}\right|, \\
\Delta & =x_{1}\left(y_{2}-y_{3}\right)+x_{2}\left(y_{3}-y_{1}\right)+x_{3}\left(y_{1}-y_{2}\right) .
\end{aligned}
$$

Likewise,

$$
\begin{aligned}
\Delta_{1} & =\frac{1}{2}\left(y_{3}-y_{2}\right)\left\{r_{a 1}^{2}-r_{a 3}^{2}+x_{3}^{2}-x_{1}^{2}+y_{3}^{2}-y_{1}^{2}\right\} \\
& -\frac{1}{2}\left(y_{3}-y_{1}\right)\left\{r_{a 2}^{2}-r_{a 3}^{2}+x_{3}^{2}-x_{2}^{2}+y_{3}^{2}-y_{2}^{2}\right\} \\
& +\frac{1}{2}\left(y_{3}-y_{2}\right)\left\{2 r_{a 1} e_{1}+e_{1}^{2}-2 r_{a 3} e_{3}-e_{3}^{2}\right\} \\
& -\frac{1}{2}\left(y_{3}-y_{1}\right)\left\{2 r_{a 2} e_{2}+e_{2}^{2}-2 r_{a 3} e_{3}-e_{3}^{2}\right\},
\end{aligned}
$$




$$
\begin{aligned}
\Delta_{2} & =\frac{1}{2}\left(x_{3}-x_{1}\right)\left\{r_{a 2}^{2}-r_{a 3}^{2}+x_{3}^{2}-x_{2}^{2}+y_{3}^{2}-y_{2}^{2}\right\} \\
& -\frac{1}{2}\left(x_{3}-x_{2}\right)\left\{r_{a 1}^{2}-r_{a 3}^{2}+x_{3}^{2}-x_{1}^{2}+y_{3}^{2}-y_{1}^{2}\right\} \\
& +\frac{1}{2}\left(x_{3}-x_{1}\right)\left\{2 r_{a 2} e_{2}+e_{2}^{2}-2 r_{a 3} e_{3}-e_{3}^{2}\right\} \\
& -\frac{1}{2}\left(x_{3}-x_{2}\right)\left\{2 r_{a 1} e_{1}+e_{1}^{2}-2 r_{a 3} e_{3}-e_{3}^{2}\right\} .
\end{aligned}
$$

From (10) and (11), the estimated value of the $x$ coordinate is given by

$$
\begin{aligned}
x & =\frac{1}{2 \Delta}\left(y_{3}-y_{2}\right)\left\{r_{a 1}^{2}-r_{a 3}^{2}+x_{3}^{2}-x_{1}^{2}+y_{3}^{2}-y_{1}^{2}\right\} \\
& -\frac{1}{2 \Delta}\left(y_{3}-y_{1}\right)\left\{r_{a 2}^{2}-r_{a 3}^{2}+x_{3}^{2}-x_{2}^{2}+y_{3}^{2}-y_{2}^{2}\right\} \\
& +\frac{1}{2 \Delta}\left(y_{3}-y_{2}\right)\left\{2 r_{a 1} e_{1}+e_{1}^{2}-2 r_{a 3} e_{3}-e_{3}^{2}\right\} \\
& -\frac{1}{2 \Delta}\left(y_{3}-y_{1}\right)\left\{2 r_{a 2} e_{2}+e_{2}^{2}-2 r_{a 3} e_{3}-e_{3}^{2}\right\} .
\end{aligned}
$$

This can be represented as

$$
x=x_{a}+E_{x} \quad \Longrightarrow \quad E_{x}=x-x_{a},
$$

where $x_{a}$ is the $x$-coordinate of the actual position of the unknown node and is given by

$$
\begin{aligned}
x_{a} & =\frac{1}{2 \Delta}\left(y_{3}-y_{2}\right)\left\{r_{a 1}^{2}-r_{a 3}^{2}+x_{3}^{2}-x_{1}^{2}+y_{3}^{2}-y_{1}^{2}\right\} \\
& -\frac{1}{2 \Delta}\left(y_{3}-y_{1}\right)\left\{r_{a 2}^{2}-r_{a 3}^{2}+x_{3}^{2}-x_{2}^{2}+y_{3}^{2}-y_{2}^{2}\right\},
\end{aligned}
$$

and $E_{x}$ is the $x$ component of the localization error:

$$
\begin{aligned}
E_{x} & =\frac{1}{2 \Delta}\left(y_{3}-y_{2}\right)\left\{2 r_{a 1} e_{1}+e_{1}^{2}-2 r_{a 3} e_{3}-e_{3}^{2}\right\} \\
& -\frac{1}{2 \Delta}\left(y_{3}-y_{1}\right)\left\{2 r_{a 2} e_{2}+e_{2}^{2}-2 r_{a 3} e_{3}-e_{3}^{2}\right\} .
\end{aligned}
$$

$x_{a}$ and $E_{x}$ can be represented using simplified notation:

$$
\begin{aligned}
& x_{a}=k_{1} C_{1}+k_{2} C_{2}, \\
& E_{x}=k_{1} E_{1}+k_{2} E_{2},
\end{aligned}
$$

where

$$
\begin{gathered}
k_{1}=\frac{1}{2 \Delta}\left(y_{3}-y_{2}\right), \\
k_{2}=-\frac{1}{2 \Delta}\left(y_{3}-y_{1}\right), \\
C_{1}=r_{a 1}^{2}-r_{a 3}^{2}+x_{3}^{2}-x_{1}^{2}+y_{3}^{2}-y_{1}^{2}, \\
C_{2}=r_{a 2}^{2}-r_{a 3}^{2}+x_{3}^{2}-x_{2}^{2}+y_{3}^{2}-y_{2}^{2}, \\
E_{1}=2 r_{a 1} e_{1}+e_{1}^{2}-2 r_{a 3} e_{3}-e_{3}^{2}, \\
E_{2}=2 r_{a 2} e_{2}+e_{2}^{2}-2 r_{a 3} e_{3}-e_{3}^{2} .
\end{gathered}
$$

$C_{1}$ and $C_{2}$ are constants because these depend upon known fixed values. However, $E_{1}$ and $E_{2}$ are variable and are functions of distance estimation errors $e_{1}, e_{2}$ and $e_{3}$.

Similarly, using (10) and (12), we get

$$
y=y_{a}+E_{y} \quad \Longrightarrow \quad E_{y}=y-y_{a},
$$

where $y_{a}$ is the $y$-coordinate of the actual position of the unknown node and is given by

$$
\begin{aligned}
y_{a} & =\frac{1}{2 \Delta}\left(x_{3}-x_{1}\right)\left\{r_{a 2}^{2}-r_{a 3}^{2}+x_{3}^{2}-x_{2}^{2}+y_{3}^{2}-y_{2}^{2}\right\} \\
& -\frac{1}{2 \Delta}\left(x_{3}-x_{2}\right)\left\{r_{a 1}^{2}-r_{a 3}^{2}+x_{3}^{2}-x_{1}^{2}+y_{3}^{2}-y_{1}^{2}\right\},
\end{aligned}
$$

and $E_{y}$ is the $y$ component of the localization error:

$$
\begin{aligned}
E_{y} & =-\frac{1}{2 \Delta}\left(x_{3}-x_{2}\right)\left\{2 r_{a 1} e_{1}+e_{1}^{2}-2 r_{a 3} e_{3}-e_{3}^{2}\right\} \\
& +\frac{1}{2 \Delta}\left(x_{3}-x_{1}\right)\left\{2 r_{a 2} e_{2}+e_{2}^{2}-2 r_{a 3} e_{3}-e_{3}^{2}\right\} .
\end{aligned}
$$

Using simplified notation:

$$
\begin{aligned}
& y_{a}=k_{3} C_{1}+k_{4} C_{2}, \\
& E_{y}=k_{3} E_{1}+k_{4} E_{2},
\end{aligned}
$$

where

$$
\begin{aligned}
k_{3} & =-\frac{1}{2 \Delta}\left(x_{3}-x_{2}\right), \\
k_{4} & =\frac{1}{2 \Delta}\left(x_{3}-x_{1}\right),
\end{aligned}
$$

$k_{1}, k_{2}, k_{3}$ and $k_{4}$ are localization constants whose values depend upon the known fixed position coordinates of beacon nodes. It is to be noted that the error variables $e_{1}, e_{2}$ and $e_{3}$ appear only in the numerators $\Delta_{1}$ and $\Delta_{2}$. There are no error terms in the denominator $\Delta$. It is further to be noted that the distance estimation error $e_{i}$ can be either positive or negative. As $r_{i}=r_{a i}+e_{i}, e_{i}$ is positive when $r_{i} \geq r_{a i}$ i.e. the estimated distance is greater than the actual distance. Similarly, $e_{i}$ is negative when $r_{i} \leq r_{a i}$ i.e. the estimated distance is smaller than the actual distance. Likewise, as is evident from (14) and (25), $E_{x}$ and $E_{y}$ can be either positive or negative. The sign gives direction of the $x$ and $y$ components of localization error.

The localization error $e_{l}$, which is only an absolute value, and is the distance between the actual $\left(x_{a}, y_{a}\right)$ and estimated $(x, y)$ positions, can now be calculated as

$$
\begin{aligned}
e_{l}= & \frac{1}{2 \Delta}\left[\left(\left(y_{3}-y_{2}\right)\left\{2 r_{a 1} e_{1}+e_{1}^{2}-2 r_{a 3} e_{3}-e_{3}^{2}\right\}\right.\right. \\
& \left.-\left(y_{3}-y_{1}\right)\left\{2 r_{a 2} e_{2}+e_{2}^{2}-2 r_{a 3} e_{3}-e_{3}^{2}\right\}\right)^{2} \\
& +\left(-\left(x_{3}-x_{2}\right)\left\{2 r_{a 1} e_{1}+e_{1}^{2}-2 r_{a 3} e_{3}-e_{3}^{2}\right\}\right. \\
& \left.\left.+\left(x_{3}-x_{1}\right)\left\{2 r_{a 2} e_{2}+e_{2}^{2}-2 r_{a 3} e_{3}-e_{3}^{2}\right\}\right)^{2}\right]^{\frac{1}{2}} .
\end{aligned}
$$

Likewise, the direction of the localization error $e_{l}$ can be determined as in (33).

\section{A. Applications of Analytical Model}

The major advantage provided by the analytical model is the isolation of localization error from the estimated location and its decomposition in its various components. As a result, the localization error and its components can be analyzed and treated using various mathematical tools which constitutes 
separate and future work. Below, we give only a few examples, where we draw important results based upon analyses of the localization error as given by the analytical model.

Corollary 1. The localization error is a function of both the distance estimation errors and the geometry of placement or topology of the reference nodes.

Proof. From (18) and (29), we infer that both $E_{x}$ and $E_{y}$ are functions of not only the distance estimation errors reflected in $E_{1}$ and $E_{2}$ but also topology of the beacon nodes as reflected in the constants $k_{1}, k_{2}, k_{3}$ and $k_{4}$. As these constants depend upon the fixed and known positions of beacon nodes, the $E_{x}$ and $E_{y}$ components of localization error also depend upon the geometry of placement or topology of the beacon nodes. Hence, the localization error is a function of both the distance estimation errors and topology of reference nodes.

We point out, it is already known that geometry of reference positions with respect to unknown node affects localization error and is described using geometric dilution of precision (GDOP) [9] in navigation. Corollary 1 corroborates this fact. This corroboration implies the correctness and usefulness of the analytical model. The model can be used to show that geometry of reference positions is significant only in the presence of distance estimation errors. If there are no distance estimation errors, there is no localization error and the topology does not have any significance.

Corollary 2. The $E_{x}$ and $E_{y}$ components of localization error are always unequal except when the neighbor beacon nodes are collinear.

Proof. From (18) and (29),

$$
E_{x}-E_{y}=\left(k_{1}-k_{3}\right) E_{1}+\left(k_{2}-k_{4}\right) E_{2} .
$$

We observe that $E_{x}=E_{y}$ if $E_{1}=E_{2}=0$ or if $k_{1}=k_{3}$ and $k_{2}=k_{4}$. However, $E_{1}=E_{2}=0$ only if there are no errors in the distance estimations i.e. $e_{1}=e_{2}=e_{3}=0$. In this case, $E_{x}=E_{y}=0$ and the localization error does not exist. On the other hand, when $k_{1}=k_{3}$ and $k_{2}=k_{4}$, then under these conditions $y_{3}-y_{2}=x_{2}-x_{3}$ and $y_{1}-y_{3}=x_{3}-x_{1}$ so that the beacon nodes are collinear and position estimation using either (5) or (6) may not be possible as the resultant matrix A is singular. In other words, the $x$ and $y$ components of localization error are always unequal except when the neighbor beacon nodes are collinear.

The analytical model of multilateration localization error developed in this section can be applied for the analysis and reduction of localization error in all engineering applications wherever multilateration is used for position estimation. This includes wireless networks, such as wireless local area networks, wireless sensor networks, Internet of things, the GNSS such as GPS and other miscellaneous industrial applications discussed in Section I. For the purpose of numerical verification of our results in the next section, we consider trilateration in wireless networks such as short range wireless sensor networks (WSN) and internet of things (IoT).

It is to note that the statistical models, such as those which use regression analysis or mean square error, are used for estimation and prediction of parameters. On the other hand, the analytical models provide their closed form expressions.

\section{NUMERICAL RESUlTS}

\section{A. Sensor Field}

We conduct a number of simulation experiments to verify the analytical model. We use a two dimensional square sensor field of size $50 \times 50$ for this purpose. In the experiments, an unknown node estimates its position with the help of three neighbor beacon nodes using trilateration. We assume that the unknown node can estimate its range $r_{i}$ from a beacon node $B_{i}$ with distance estimation error $e_{i}$.

\section{B. Experiments}

To verify the analytical model of localization error developed in Section III, we run two types of simulation experiments. The experiments use an unknown node $U_{1}$ positioned at $(30,20)$ with three neighbor beacon nodes $B_{1}$, $B_{2}$ and $B_{3}$. Five experiments of each of these two types are carried out. We choose this simple configuration as the multilateration solution and the model are analytical, closed form and deterministic.

1) Type I Experiments: In the first set of experiments, the positions of the three beacon nodes and hence their actual distances from the unknown node remain fixed and these test settings are recorded in Table I. In this way, the topology of the nodes remains unchanged with each experiment in the first set. However, we introduce a variable range estimation error with each experiment so that the estimated distances are different for each experiment in the first set of experiments. Summary of results of first type of experiments is given in Table III.

2) Type II Experiments: In the second set of the experiments, we change the positions of nodes and hence the resulting topology with each experiment but use the same set of distance estimation errors and estimated distances. For this purpose, we vary the positions of the beacon nodes such that magnitudes of their distances from the unknown node remain unchanged. In each experiment in the second set of experiments, we pick new position of a beacon node along the circumference of the circle with unknown node as the center and its fixed distance from the beacon node as radius. In this way, the topology of nodes changes with each new experiment in the second set. However, the estimated distances and the distance estimation errors remain unchanged. These

$$
\theta_{l}=\tan ^{-1} \frac{-\left(x_{3}-x_{2}\right)\left\{2 r_{a 1} e_{1}+e_{1}^{2}-2 r_{a 3} e_{3}-e_{3}^{2}\right\}+\left(x_{3}-x_{1}\right)\left\{2 r_{a 2} e_{2}+e_{2}^{2}-2 r_{a 3} e_{3}-e_{3}^{2}\right\}}{\left(y_{3}-y_{2}\right)\left\{2 r_{a 1} e_{1}+e_{1}^{2}-2 r_{a 3} e_{3}-e_{3}^{2}\right\}-\left(y_{3}-y_{1}\right)\left\{2 r_{a 2} e_{2}+e_{2}^{2}-2 r_{a 3} e_{3}-e_{3}^{2}\right\}}
$$




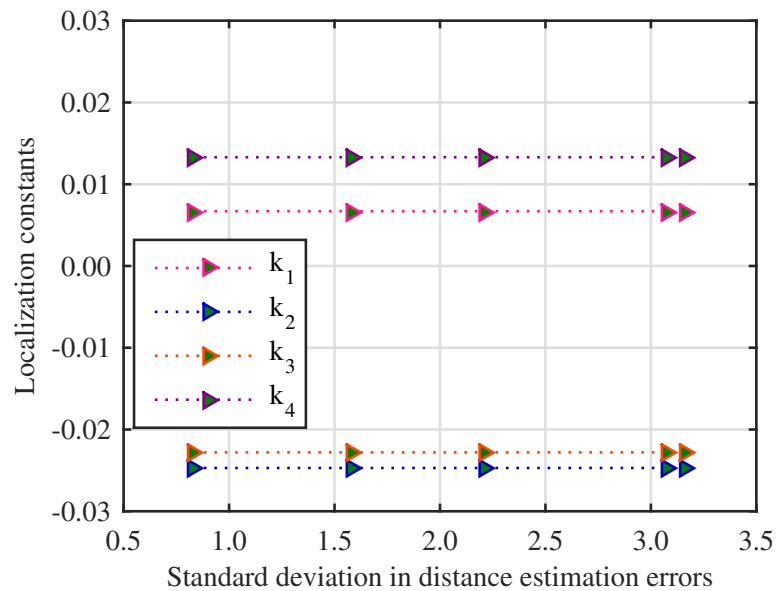

Fig. 1. Localization constants when the positions of beacon nodes are fixed with variable distance estimation errors.

TABLE I

Positions and Distances of Beacon Nodes in a Fixed Topology.

\begin{tabular}{ccc}
\hline $\begin{array}{c}\text { Beacon } \\
\text { Node }\end{array}$ & Position & $\begin{array}{c}\text { Actual Distance } \\
\text { from Unknown Node }\end{array}$ \\
\hline$B_{1}$ & $(30,34)$ & 14.00 \\
\hline$B_{2}$ & $(40,15)$ & 11.18 \\
\hline$B_{3}$ & $(16,8)$ & 18.44 \\
\hline
\end{tabular}

TABLE II

FiXed Distances of Beacon Nodes in a VARIABle Topology.

\begin{tabular}{cccc}
\hline $\begin{array}{c}\text { Beacon } \\
\text { Node }\end{array}$ & $\begin{array}{c}\text { Actual Distance } \\
\text { from Unknown Node }\end{array}$ & $\begin{array}{c}\text { Distance } \\
\text { Estimation Error }\end{array}$ & $\begin{array}{c}\text { Estimated } \\
\text { Distance }\end{array}$ \\
\hline$B_{1}$ & 14.00 & 0.13 & 14.13 \\
\hline$B_{2}$ & 11.18 & -0.40 & 10.79 \\
\hline$B_{3}$ & 18.44 & -1.50 & 16.94 \\
\hline
\end{tabular}

test settings are given in Table II. Summary of results of second type of experiments is given in Table IV.

\section{Localization Constants}

Localization constants are plotted in Fig. 1 when distance estimation errors are varied while the positions and hence topology of the beacon nodes is fixed. These constants are again plotted in Fig. 2 when the positions of beacon nodes are changed while the distance estimation errors are kept constant. It is evident from Fig. 1 and Fig. 2 that the localization constants remain the same for a given topology of beacon nodes and only vary with positions and hence topology of the beacon nodes. Moreover, these are unaffected by the distance estimation errors. In other words, any change in topology of beacon nodes is reflected by a change in localization constants. Furthermore, the localization constants are only functions of topology of beacon nodes and are independent of distance estimation errors.

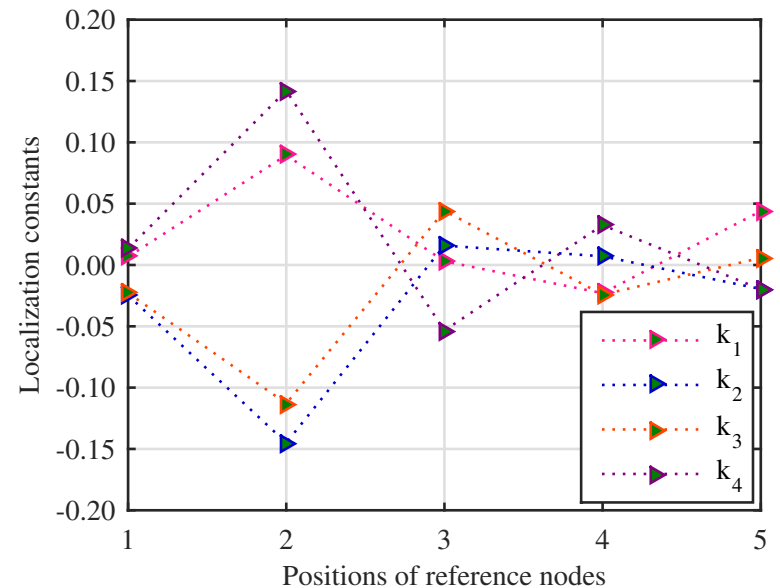

Fig. 2. Localization constants when the positions of beacon nodes are varied with fixed distance estimation errors.

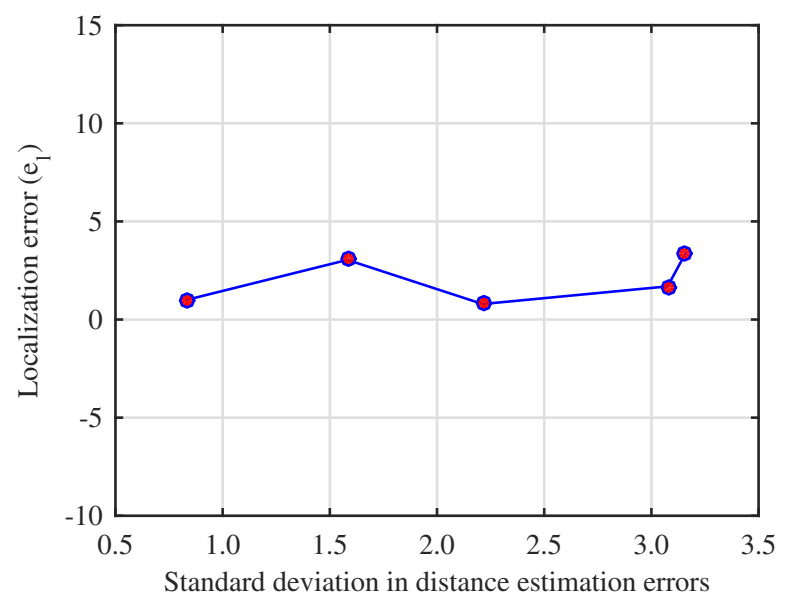

Fig. 3. Localization error $e_{l}$ when the positions of beacon nodes are fixed with variable distance estimation errors.

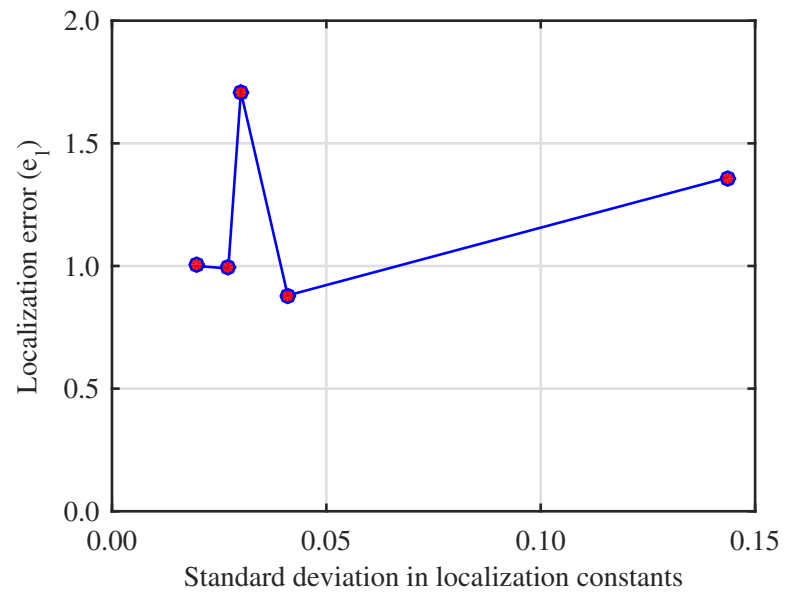

Fig. 4. Localization error $e_{l}$ when the positions of beacon nodes are varied while keeping fixed distance estimation errors.

\section{Localization Error $\left(e_{l}\right)$}

The localization error $e_{l}$ is plotted against standard deviation in distance estimation errors in Fig. 3 when the positions of 
TABLE III

Positions of Beacon Nodes are Fixed but Distance Estimation Errors are Variable.

\begin{tabular}{|c|c|c|c|c|c|c|c|c|c|c|c|c|c|c|c|c|}
\hline \multirow[t]{2}{*}{$\begin{array}{l}\text { Exp. } \\
\text { No. }\end{array}$} & \multicolumn{3}{|c|}{$\begin{array}{c}\text { Distance } \\
\text { Estimation } \\
\text { Error }\end{array}$} & \multicolumn{3}{|c|}{$\begin{array}{l}\text { Estimated } \\
\text { Distances }\end{array}$} & \multirow{2}{*}{$\begin{array}{c}\text { Experimental } \\
\text { Results } \\
(x, y) \\
\end{array}$} & \multicolumn{6}{|c|}{ Analytical Results } & \multicolumn{3}{|c|}{ Common Results } \\
\hline & $e_{1}$ & $e_{2}$ & $e_{3}$ & $r_{1}$ & $r_{2}$ & $r_{3}$ & & $k_{1}$ & $k_{2}$ & $k_{3}$ & $k_{4}$ & $E_{1}$ & $E_{2}$ & $E_{x}$ & $E_{y}$ & $e_{l}$ \\
\hline 1 & 0.13 & -0.40 & -1.50 & 14.13 & 10.79 & 16.94 & $(29.28,19.30)$ & 0.0067 & -0.0247 & -0.0228 & 0.0133 & 56.56 & 44.25 & -0.72 & -0.70 & 1.00 \\
\hline 2 & -4.05 & -0.66 & 2.09 & 9.95 & 10.52 & 20.53 & $(31.18,22.80)$ & 0.0067 & -0.0247 & -0.0228 & 0.0133 & -178.53 & -95.93 & 1.18 & 2.80 & 3.04 \\
\hline 3 & -3.84 & -4.22 & -1.31 & 10.16 & 6.96 & 17.13 & $(30.43,20.66)$ & 0.0067 & -0.0247 & -0.0228 & 0.0133 & -46.27 & -30.03 & 0.43 & 0.66 & 0.79 \\
\hline 4 & -4.66 & -3.08 & -0.29 & 9.34 & 8.10 & 18.15 & $(30.55,21.59)$ & 0.0067 & -0.0247 & -0.0228 & 0.0133 & -98.35 & -48.88 & 0.55 & 1.59 & 1.69 \\
\hline 5 & -3.55 & 2.18 & 1.62 & 10.45 & 13.36 & 20.06 & $(29.23,23.28)$ & 0.0067 & -0.0247 & -0.0228 & 0.0133 & -149.07 & -8.80 & -0.77 & 3.28 & 3.37 \\
\hline
\end{tabular}

TABLE IV

Positions of Beacon Nodes are Variable but Distance Estimation Errors are Fixed.

\begin{tabular}{|c|c|c|c|c|c|c|c|c|c|c|c|c|c|}
\hline \multirow[t]{2}{*}{$\begin{array}{l}\operatorname{Exp} \\
\text { No. }\end{array}$} & \multicolumn{3}{|c|}{$\begin{array}{c}\text { Beacon Node } \\
\text { Position } \\
\end{array}$} & \multirow{2}{*}{$\begin{array}{c}\begin{array}{c}\text { Experimental } \\
\text { Results }\end{array} \\
(x, y) \\
\end{array}$} & \multicolumn{6}{|c|}{ Analytical Results } & \multicolumn{3}{|c|}{ Common Results } \\
\hline & $B_{1}$ & $B_{2}$ & $B_{3}$ & & $k_{1}$ & $k_{2}$ & $k_{3}$ & $k_{4}$ & $E_{1}$ & $E_{2}$ & $E_{x}$ & $E_{y}$ & $e_{l}$ \\
\hline 1 & $(30.00,34.00)$ & $(40.00,15.00)$ & $(16.00,8.00)$ & $(29.28,19.30)$ & 0.0067 & -0.0247 & -0.0228 & 0.0133 & 56.56 & 44.25 & -0.72 & -0.70 & 1.00 \\
\hline 2 & $(43.82,22.26)$ & $(39.83,14.67)$ & $(24.39,2.44)$ & $(28.65,19.90)$ & 0.0895 & -0.1450 & -0.1129 & 0.1421 & 56.56 & 44.25 & -1.35 & -0.10 & 1.36 \\
\hline 3 & $(16.41,16.63)$ & $(21.78,27.58)$ & $(47.49,25.83)$ & $(30.87,20.14)$ & 0.0030 & 0.0158 & 0.0442 & -00524 & 56.56 & 44.25 & 0.87 & 0.14 & 0.88 \\
\hline 4 & $(43.27,24.46)$ & $(36.11,10.63)$ & $(14.98,30.69)$ & $(29.01,20.07)$ & -0.0230 & 0.0071 & -0.0242 & 0.0325 & 56.56 & 44.25 & -0.99 & 0.07 & 0.99 \\
\hline 5 & $(17.14,14.48)$ & $(26.38,30.58)$ & $(30.23,1.56)$ & $(31.62,19.45)$ & 0.0439 & -0.0196 & 0.0058 & -0.0198 & 56.56 & 44.25 & 1.62 & -0.55 & 1.71 \\
\hline
\end{tabular}

beacon nodes are kept fixed and it is plotted against standard deviation in localization constants in Fig. 4 when the distance estimation errors are fixed. The change in localization error with each iteration in the former case is contributed by the distance estimation errors alone and by the change in topology of reference nodes alone in the latter case. Therefore, change in the localization error from one iteration to the next is a function of both the distance estimation errors and the topology of the reference nodes as is also concluded using the analytical model in Section III.

\section{CONCLUSION}

We have presented an accurate analytical model for trilateration error. The model isolates the localization error from the estimated position and decomposes it into various components. This allows treatment of localization error by using various mathematical techniques. Therefore, the analytical model can be used for the investigation and analysis of localization error due to trilateration with inaccurate range estimates. As an example application, we have used the analytical model to corroborate the known fact that topology of beacon nodes along with distance estimation errors affects and contributes to the localization error. Therefore, the localization error varies with change in topology of beacon nodes even when the distance estimation errors remain unchanged. The proposed model can be used for the analysis and reduction of localization error in a wide range of engineering applications. All our analytical results have been verified using simulation.

\section{REFERENCES}

[1] H. Wang, L. Wan, M. Dong, K. Ota, and X. Wang, "Assistant vehicle localization based on three collaborative base stations via SBL-based robust DOA estimation," IEEE Internet of Things Journal, vol. 6, no. 3, pp. 5766-5777, June 2019.

[2] J. Fan, Y. Hu, T. H. Luan, and M. Dong, "DisLoc: A convex partitioning based approach for distributed 3-D localization in wireless sensor networks," IEEE Sensors Journal, vol. 17, no. 24, pp. 8412-8423, Dec 2017.

[3] M. Farooq-I-Azam, Q. Ni, and E. A. Ansari, "Intelligent energy efficient localization using variable range beacons in industrial wireless sensor networks," IEEE Transactions on Industrial Informatics, vol. 12, no. 6 , pp. 2206-2216, Dec 2016.

[4] B. Li, S. Li, A. Nallanathan, and C. Zhao, "Deep sensing for future spectrum and location awareness 5G communications," IEEE Journal on Selected Areas in Communications, vol. 33, no. 7, pp. 1331-1344, July 2015.

[5] M. Farooq-I-Azam, W. Yu, Q. Ni, M. Dong, and A. ul Quddus, "Location assisted subcarrier and power allocation in underlay mobile cognitive radio networks," in 2018 IEEE Globecom Workshops (GC Wkshps), Dec 2018, pp. 1-6.

[6] "Facilitating opportunities for flexible, efficient, and reliable spectrum use employing cognitive radio technologies," Federal Communications Commission, Tech. Rep. FCC-05-57, March 2005.

[7] F. Chiti, R. Fantacci, F. Archetti, E. Messina, and D. Toscani, "An integrated communications framework for context aware continuous monitoring with body sensor networks," IEEE Journal on Selected Areas in Communications, vol. 27, no. 4, pp. 379-386, May 2009.

[8] A. Giannitrapani, N. Ceccarelli, F. Scortecci, and A. Garulli, "Comparison of EKF and UKF for spacecraft localization via angle measurements," IEEE Transactions on Aerospace and Electronic Systems, vol. 47, no. 1, pp. 75-84, January 2011.

[9] D. J. Torrieri, "Statistical theory of passive location systems," IEEE Transactions on Aerospace and Electronic Systems, vol. AES-20, no. 2, pp. 183-198, March 1984. 